\title{
FRACTURE RESISTANCE ASSESSMENT OF ENDODONTICALLY ACCESSED HYBRID CERAMIC CROWNS REPAIRED WITH TWO DIFFERENT PROTOCOLS
}

\author{
Sherif El Khodary* and Reham El Basty**
}

\begin{abstract}
Statement of the Problem: It is frustrating when the patient has recently placed an all ceramic crown and now has symptoms of irreversible pulpitis or necrosis and an endodontic treatment is indicated. Providing endodontic care through all ceramic crowns in situ is a challenge. Endodontic access through the restoration may weaken it and initiate micro cracks that may propagate resulting in failure. The best practice protocol to improve the fracture resistance of endodontically accessed and repaired all ceramic crowns cannot be identified based on the currently available scientific data. In addition, wider variations in repair protocols are worthy of further investigation.
\end{abstract}

Purpose: The aim of this in vitro study was to measure the fracture resistance of endodontically accessed Vita Enamic crowns repaired with two different protocols (direct composite and ceramic plug).

Materials and Methods: Fifteen freshly extracted human mandibular molar teeth were used in this study. All teeth were mounted in epoxy resin blocks using a special device (parallelometer). Teeth were randomly divided into three groups of five specimens each according to the type of restoration as follows; Group I: Teeth restored with full coverage CAD/CAM Enamic crowns (control group), Group II: Teeth restored with full coverage CAD/CAM Enamic crowns, endodontically treated and repaired with direct composite, Group III: Teeth restored with full coverage CAD/ CAM Enamic crowns, endodontically treated and repaired with CAD/CAM bonded enamic plugs. Teeth were prepared using a special milling machine. Cerec Premium SW 4.4 was used for the fabrication of crowns from hybrid ceramic Enamic blocks. Crowns were cemented using Rely X Ultimate dual-cure resin cement. For groups II and III, an access cavity was made at the center of the occlusal surface using a round-end diamond bur and copious amounts of water spray followed by root canal treatment. A thin layer of flowable composite material was placed at the base of the pulp chamber. For group II; the access cavity was filled with Filtek Supreme nanofilled composite. For group III; the access cavity was scanned then a ceramic plug filling the access cavity was designed using the Cerec Premium software and milled from Vita Enamic blocks. The ceramic plug was then cemented inside the access cavity using Rely X Ultimate resin cement. All the specimens were subjected to thermo mechanical aging in a chewing simulator for 150,000 cycles.

* Lecturer, Endodontic Department, Faculty of Dentistry. Cairo University.

** Lecturer, Fixed Prosthodontics Department, Faculty of Dentistry. Cairo University. 
Fracture resistance was measured using a computer controlled universal testing machine. For each crown, a single static compressive load was applied along the long axis till fracture occurred. The load required to induce failure was recorded in Newton (N).Data were recorded, tabulated and statistically analyzed.

Results: The highest mean \pm SD values were recorded for group I $(2027.64 \pm 294.24 \mathrm{~N})$ followed by group III $(1784.26 \pm 72.15 \mathrm{~N})$ while the lowest mean $\pm \mathrm{SD}$ values were recorded for group II $(1494.95 \pm 46.95 \mathrm{~N})$. The difference between groups was statistically significant as indicated by ANOVA test $(\mathrm{p}=0.0017<0.05)$. Pair-wise Tukey's post-hoc tests showed non-significant $(\mathrm{p}>0.05)$ differences between (group I and group III) and (group II and group III).

Conclusions : 1- The endodontically accessed hybrid ceramic crowns repaired with the novel protocol (ceramic plug) recorded high fracture resistance values. 2- The use of ceramic plug is a feasible alternative to the currently used composite repair protocol.

\section{INTRODUCTION}

Dental crowns are distinguished to be a durable and uncomplicated option while at the same time generating the highest income ${ }^{(1)}$. However, crown preparation is irreversibly destructive to the tooth. Typically $62-73 \%$ of tooth structure is removed during all ceramic crown preparation ${ }^{(2)}$. The relation between tooth destruction and possible pulpal complications is documented in the literature ${ }^{(2,3,4,5)}$. Despite this, the decision to perform a precautionary root canal prior to crown construction because there is the lingering fear that a root canal may be necessary sometime down the road is not easy for both the dentist and the patient.

Goodacre et al. ${ }^{(6)}$ explored the incidence of clinical complications of dental crowns over a 50 year period and identified that $3 \%$ of crowns required subsequent endodontic therapy. A metaanalysis of 34 studies within a 5 year period showed $2.1 \%$ incidence of loss of vitality after all ceramic crown placement. ${ }^{(7)}$ Recent studies highlighted re intervention for all ceramic crowns requiring endodontic therapy of $4 \%$ and $8.6 \%$ for five ${ }^{(8)}$ and seven ${ }^{(9)}$ year follow up intervals.

Although low incidences of re intervention has been reported, it has been postulated that the actual incidences of pulpal complications may be under recorded when determination has been made only through clinical assessment ${ }^{(10)}$ and not through radiographic evidence. ${ }^{(11)}$ Saunders and Saunders ${ }^{(11)}$ declared a high incidence of pulpal complications (19\%) through periapical radiographs in crowned teeth confirming the asymptomatic potential of pulpal complications.

The need to save rather than replace a crown is a financial issue for many patients and one that is desirable for the dentist especially if the crown was new. Removing a crown before root canal treatment is performed and re-cementing it afterwards would be the ideal treatment plan. Unfortunately, crown can be extremely difficult to remove and it is possible that it will be damaged during the process. Moreover, the tooth itself might be damaged. Also, most of the time, all ceramic crowns are bonded using resin cements which render the safe removal of the crowns impossible, making the solution of access preparation through the bonded crowns inevitable. ${ }^{(12)}$

However, endodontic therapy through an existing crown has 2 main challenges; first, access orientation is difficult because the crown covers the coronal tooth structure, so dentists must use their best judgment to determine the location of the pulp chamber, second, the clinician must determine how to manage the access opening. A restorative material that prevents marginal and intermaterial leakage 
and restores the fracture resistance of the existing crown should be used. ${ }^{(13)}$

All-ceramic crowns have additional challenges when endodontic treatment is indicated. Ceramics have poor thermal conductivity so it is difficult to control heat formation during access opening ${ }^{(13)}$. Also, their fracture toughness is flaw dependent and they are brittle materials ${ }^{(14,15)}$. Flaws such as microcracks can be initiated as a result of preparation of endodontic access openings through the existing crown and thus negatively affecting the mechanical properties of the restoration.

According to Gorman et al. in $2016^{(16)}$, the key factors which would influence the fracture resistance of endodontically accessed and repaired all ceramic crowns are; the material from which the crown has been made, its adhesive potential and its initial baseline strength, whether the crown has been adhesively or non-adhesively bonded, the grit size of the diamond selected to create the access cavity, the ratio of endodontic access cavity to the crown size and evaluating the access cavity for proceeding to repair or even remake of the crown. If the structural integrity of the crown has been compromised and it can no longer be relied upon to protect the tooth it has to be replaced. However, if the structural integrity seems reasonable, the clinician will complete the crown repair. ${ }^{(12)}$

Another important factor affecting the fracture resistance of endodontically accessed and repaired all ceramic crowns is the material from which the access is repaired. Access cavity is routinely repaired using direct resin composites providing a very cost-effective solution ${ }^{(17)}$. However, if the material placed is not strong enough or does not provide a coronal seal to block the seepage of contaminants back into the tooth, the longevity of the repaired crown can be jeopardized ${ }^{(18)}$

A new approach to repair the access cavity is using a ceramic plug that is made from the same crown material and bonded to both the tooth structure and the existing crown.
Therefore, the aim of this study was to measure the fracture resistance of endodontically accessed hybrid ceramic crowns repaired with either direct composite or a bonded ceramic plug. The null hypothesis is that the bonded ceramic plug would not increase the fracture resistance values of repaired endodontically accessed hybrid ceramic crowns compared to direct composite resin.

\section{MATERIALS AND METHODS}

Fifteen freshly extracted human mandibular molar teeth, free of caries or fractures were used in this study. The anatomic crowns were confirmed to be with average dimensions after measuring the bucco-lingual and mesio-distal widths using a digital caliper at the level of the cementoenamel junction. Teeth were cleaned with an ultrasonic scaler and under copious water coolant and were then kept in distilled water at room temperature prior to the study. All teeth were mounted in epoxy resin blocks $(2 \times 2 \times 2 \mathrm{~cm})$ using a special device (parallelometer) to allow accurate vertical centralization of the teeth in the block ; with the long axis of each tooth parallel to the long axis of the block.

\section{Teeth Grouping}

Teeth were randomly divided into three groups of five specimens each according to the type of restoration as follows; Group I: Teeth restored with full coverage CAD/CAM Enamic crowns (control group), Group II: Teeth restored with full coverage CAD/CAM Enamic crowns, endodontically treated and repaired with direct composite, Group III: Teeth restored with full coverage CAD/CAM Enamic crowns, endodontically treated and repaired with CAD/CAM bonded enamic plugs.

Before any intervention, all teeth were scanned using Omnicam intra-oral scanner (Sirona Dental System, Benshein, Germany) for the unprepared anatomy to be used in the fabrication of the artificial ceramic crowns. The virtual images obtained were archived in the Biogeneric copy catalogue of the software. Teeth were prepared using a special 
milling machine to produce a preparation with a 6 degrees axial taper, an occluso cervical length of $5 \mathrm{~mm}$ and a $0.8 \mathrm{~mm}$ deep chamfer finish line.

\section{Crown construction}

A CAD/CAM system (Cerec Premium SW 4.4) (Sirona Dental System, Benshein, Germany) was used for the fabrication of crowns in this study from hybrid ceramic Enamic blocks (Vita Zahnfabrik, Germany). Optical scanning of each prepared tooth was made with the Omnicam intra-oral scanner and a 3 dimensional image was obtained for each prepared tooth. With the aid of Cerec Premium software and the biogeneric copy option the scanned unprepared tooth was correlated to the prepared tooth to fabricate a crown that is identical to the tooth form before preparation. Then milling was performed using Cerec InLab MCXL 4-axis milling machine (Sirona Dental System, Benshein, Germany). Milled crowns were checked over their corresponding teeth for seating, then finished and polished using Vita Enamic polishing set (Vita Zahnfabrik, Germany) according to manufacturer's instructions.

\section{Cementation Procedures}

Prior to cementation, the intaglio surfaces of the crowns were cleaned with alcohol and air dried. A 5\% hydrofluoric acid (IPS Ceramic Refill, Ivoclar Vivadent, Schaan, Liechtenstein) was used for etching the fitting surfaces for 60 seconds according to manufacturer's instructions. The Etched surfaces were then rinsed with water spray for 60 seconds and air dried for another 60 seconds. This was followed by the application of silane coupling agent (RelyX Ceramic Primer, 3M ESPE Dental products, St. Paul,USA) which was left to dry for 60 seconds. Then, two coats of bonding agent (Adper Single bond 2 adhesive, 3M ESPE Dental products, St. Paul, USA) were applied on the fitting and left without light curing so as not to interfere with restoration seating. Etching of the prepared tooth was done using 37\% phosphoric acid for 30 seconds (Scotchbond etchant, 3M ESPE Dental products, St. Paul,
USA) then the acid was rinsed and dried. Bonding agent Adper Single bond 2 adhesive was then applied to the tooth and light polymerized for 10 seconds. Crowns were cemented using Rely X U1timate dual-cure resin cement (3M ESPE, Seefeld, Germany). Cement was mixed, applied to the inner surface of the crown and each crown was seated on its respective tooth with finger pressure. Excess cement was carefully removed from the margins after a preliminary 5 seconds light curing. Then a customized loading device was used to apply an axial load of $3 \mathrm{~kg}$ to prevent rebounding of the crown during cementation. The cement was light cured for 40 seconds from all surfaces then teeth were stored in saline at room temperature until testing.

\section{Endodontic treatment}

For groups II and III, an access cavity was made at the center of the occlusal surface of the crown using a high speed round-end diamond bur and copious amounts of water spray. Endodontic procedures for all teeth were performed by the same operator and kept as conservative as possible allowing an unobstructed access to the apex.

Root canal preparation was carried out as follows; canals were accessed and canal patency was confirmed with a size $10 \mathrm{~K}$-file (Dentsply Maillefer, Ballaigues, Switzerland). Working length was determined using digital radiography. The glide path was expanded using PathFiles P1 and P2 as recommended by the manufacturer's instructions.

ProTaper Next was used with a 16:1 gear reduction electric handpiece (X-SMART plus, endodontic motor; Dentsply Maillefer, Ballaigues, Switzerland) at $300 \mathrm{rpm}$ and 2.0 torque.

In the presence of sodium hypochlorite $(\mathrm{NaOCl})$, the rotary files were introduced into the canals using EDTA gel (MD-Chelcream, META BIOMED CO.,LTD, Korea). The X1 (17/04) and X2 (25/06) files were used in pecking motion until working length was reached in the mesiobuccal and mesiolingual canals. X3 (30/07) and X4 (040/06) 
files were used for the distal canals as for X1 and X2 files. The canals were irrigated with $2 \mathrm{ml} 2 \% \mathrm{NaOCl}$ between each file using a 27 gauge needle and finally the canals were flushed with $2 \mathrm{ml} 17 \%$ EDTA. The canals were then dried using paper points and the corresponding gutta percha points were checked to reach the full working length. Obturation was done using lateral condensation technique using resin sealer (ADSEAL, META BIOMED CO., LTD, Korea).

A thin layer of flowable composite material (Filtek Z350, 3M ESPE Dental products, St. Paul, USA) was placed at the base of the pulp chamber after obturation to seal the canals entrance and provide a flat base. Fig. (1)

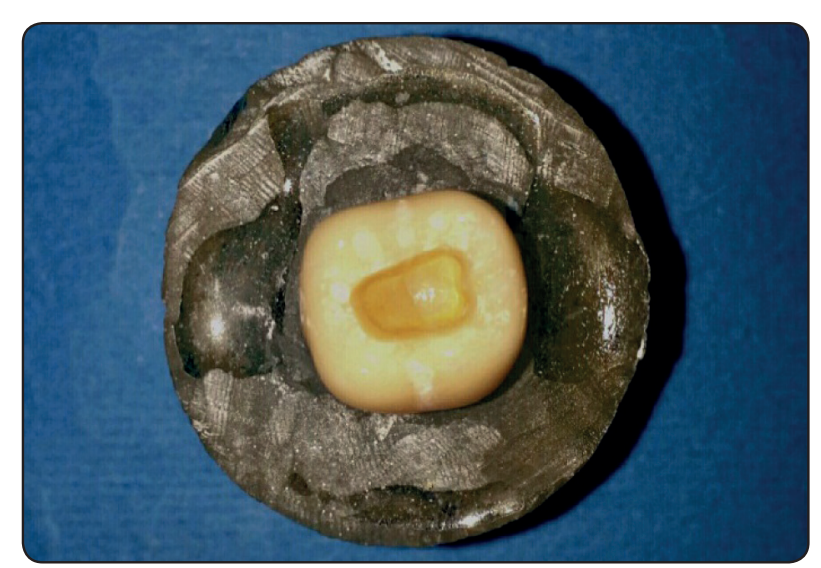

Fig. (1) Endodontic access through Enamic crown.

\section{Access Repair}

Alcohol was used to clean the inner surfaces of the access cavity. The dentin surface was refreshed with a bur and then etched with $37 \%$ phosphoric acid for 30 seconds. The acid was rinsed then the cavity was dried. Silane coupling agent (RelyX Ceramic Primer) was applied to the porcelain and allowed to dry for 60 seconds. Then adper single bond 2 dentin bonding agent was applied to both dentin and porcelain and light polymerized for 10 seconds.

For group II; the access cavity was filled with Filtek Supreme nanofilled composite (3M ESPE
Dental products, St. Paul, USA). Composite was finished with a rubber wheel under water irrigation.

For group III; the access cavity was scanned using Omnicam intra oral scanner to obtain a three dimensional image of the access cavity on the computer screen. Then a ceramic plug filling the access cavity was designed using the Cerec Premium software and milled from Vita Enamic blocks. Fig. (2) Milled plugs were placed inside the access cavity and checked for seating. Fig. (3)

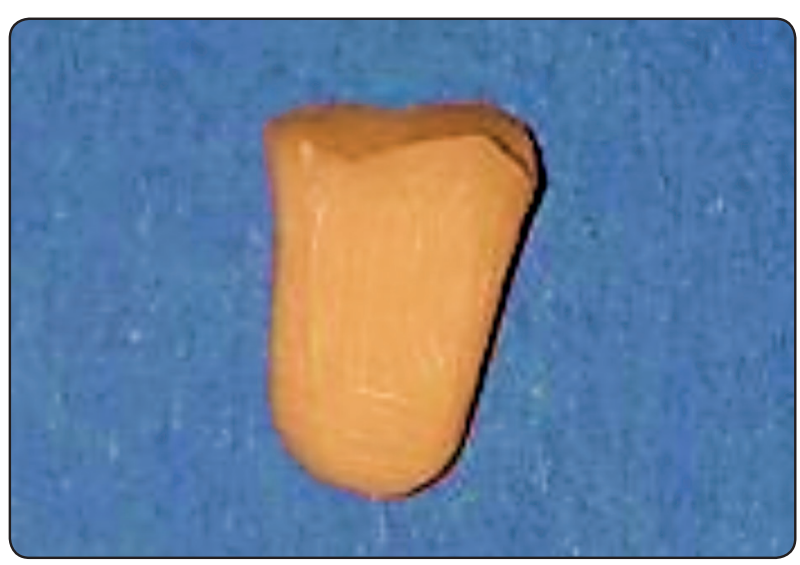

Fig. (2) Milled Enamic plug.

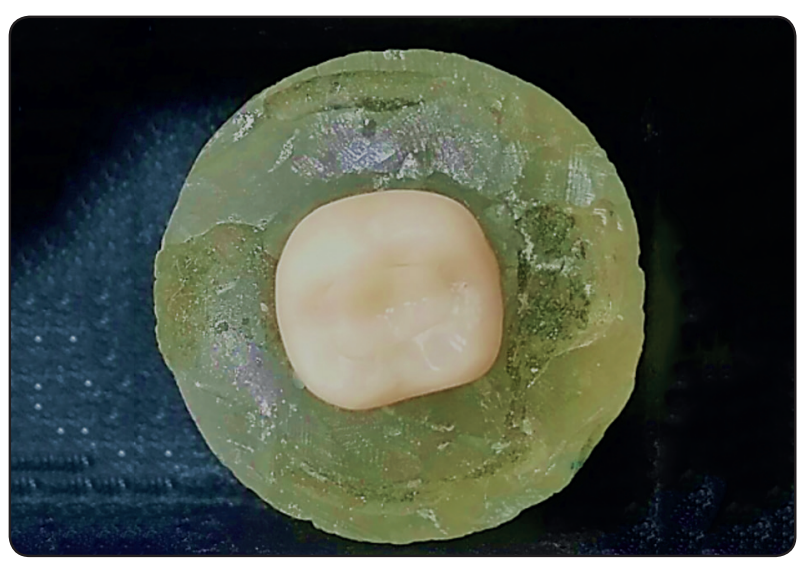

Fig. (3) Enamic Plug seated inside the access cavity

The ceramic plug was then etched and silanized with the same protocol mentioned before, then cemented inside the access cavity using Rely $\mathrm{X}$ Ultimate resin cement. The cement was light polymerized for 60 seconds to ensure adequate polymerization in deep areas. After cementation, 
the plugs were finished and polished using Vita Enamic polishing set according to manufacturer's instructions.

\section{Thermo mechanical aging:}

All crowns in the three groups were subjected to thermo mechanical aging using the newly developed four stations multi-model Robota chewing simulator (Model ACH-09075DC-T, ADTECH Technology Co., LTD., Germany). Robota chewing simulator has four chambers simulating horizontal and vertical movements simultaneously. Every chamber consists of an upper hardened steel stylus holder tightened with a screw to be used as an antagonist and a lower plastic sample holder where the specimens were embedded in a water bath. A $5 \mathrm{Kg}$ weight comparable to $49 \mathrm{~N}$ of a chewing force was applied. The test was repeated for 150,000 cycles. Chewing simulation test parameters were summarized in table 1:

TABLE (1): Chewing simulation test parameters

\begin{tabular}{|c|c|}
\hline $\begin{array}{c}\text { Cold/Hot bath temperature: } \\
5{ }^{\circ} \mathrm{C} / 55^{\circ} \mathrm{C}\end{array}$ & Dwell time: $60 \mathrm{~s}$ \\
\hline Vertical movement: $2 \mathrm{~mm}$ & Horizontal movement: $3 \mathrm{~mm}$ \\
\hline Rising speed: $90 \mathrm{~mm} / \mathrm{s}$ & Forward speed: $90 \mathrm{~mm} / \mathrm{s}$ \\
\hline Descending speed: $40 \mathrm{~mm} / \mathrm{s}$ & Backward speed: $40 \mathrm{~mm} / \mathrm{s}$ \\
\hline Cycle frequency: $3 \mathrm{~Hz}$ & Weight per sample: $5 \mathrm{Kg}$ \\
\hline Torque: $2.4 \mathrm{~N} . \mathrm{m}$ & \\
\hline
\end{tabular}

\section{Fracture resistance measurement}

The epoxy resin blocks were mounted in the lower compartment of a computer controlled universal testing machine (Instron 6022, Instron Limited, High Wycombe, UK). For each crown, a single static compressive load was applied along the long axis till fracture occurred. The load was applied using a load applicator in the form of a steel rod with a rounded tip ( $4 \mathrm{~mm}$ in diameter) and centered on the occlusal surface in three contact points. A rubber dam sheet was placed on the occlusal surface to allow even distribution of the load. The load required to induce failure was recorded in Newton (N) for each specimen using a computer software.

Data were recorded, tabulated and statistically analyzed using One-way analysis of variance with the aid of a computer software.

\section{RESULTS}

Data analysis was performed in several steps. Initially, descriptive statistics for each group was done. One way ANOVA-test followed by pair-wise Tukey's post-hoc test was done between groups. Statistical analysis was performed using GraphPad Instat statistics software for Windows (www. graphpad.com). $\mathrm{P}$ values $\leq 0.05$ are considered to be statistically significant.

Descriptive statistics of fracture resistance test results; mean values, standard deviation (SD) and confidence intervals (low and high) for all groups were summarized in table (2) and graphically drawn in figure (4)

The highest mean $\pm \mathrm{SD}$ values were recorded for group I "Teeth restored with full coverage Enamic crowns (control group)" (2027.64 \pm 294.24 N) followed by group III "Teeth restored with full coverage Enamic crowns, endodontically treated and repaired with bonded Enamic plugs" $(1784.26 \pm 72.15 \mathrm{~N})$ while the lowest mean \pm SD values were recorded for group II "Teeth restored with full coverage Enamic crowns, endodontically treated and repaired with direct composite" $(1494.95 \pm 46.95 \mathrm{~N})$. The difference between groups was statistically significant as indicated by ANOVA test $(\mathrm{p}=0.0017<0.05)$.

Pair-wise Tukey's post-hoc tests showed nonsignificant $(\mathrm{p}>0.05)$ differences between (group I and group III) and (group II and group III) 


\section{Mode of failure assessment :}

Visually, all the crowns in the three groups exhibited longitudinal crown fracture into several segments with the majority of the crown remained bonded to the tooth, while the prepared tooth structure remained intact. Fig. (5)

TABLE (2) Descriptive statistics of fracture resistance test results (Mean values \pm SD) for all groups

\begin{tabular}{|c|c|c|c|c|c|c|c|}
\hline \multirow{2}{*}{ Variables } & \multirow{2}{*}{ Mean } & \multirow{2}{*}{$\begin{array}{c}\text { SD } \\
\text { Low }\end{array}$} & & \multicolumn{2}{c|}{ CI } & \multicolumn{2}{c|}{ ANOVA } \\
\cline { 5 - 9 } & & & High & F & P value & \\
\hline \multirow{3}{*}{$\begin{array}{c}\text { Experimental } \\
\text { Group }\end{array}$} & Group I & $2027.64^{\mathrm{A}}$ & 294.24 & 1662.4 & 2392 & \multirow{2}{*}{11.4} & \multirow{2}{*}{$0.0017^{*}$} \\
\cline { 2 - 6 } & Group II & $1494.95^{\mathrm{B}}$ & 46.95 & 1436.7 & 1553.2 & & \\
\cline { 2 - 7 } & Group III & $1784.26^{\mathrm{AB}}$ & 72.15 & 1694.3 & 1873.8 & & \\
\hline
\end{tabular}

Different letters in the same column indicating statistically significant difference $(p<0.05)$

*; significant $(p<0.05)$

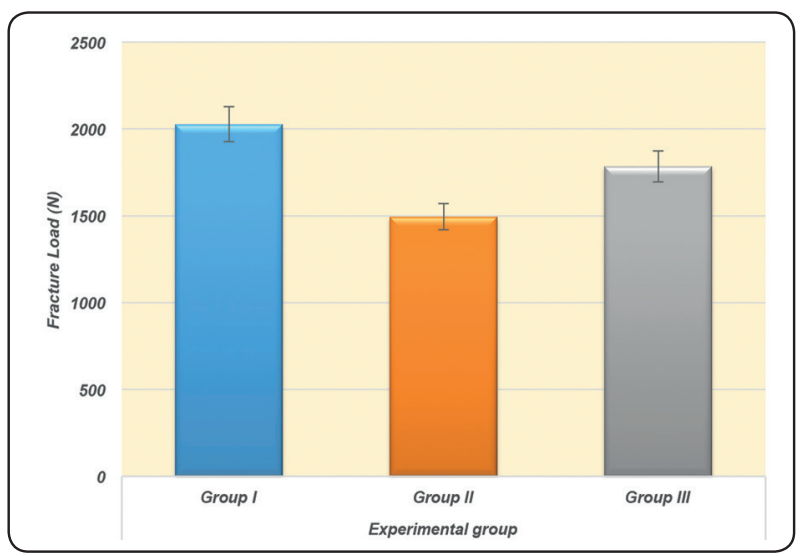

Fig. (4) Column chart showing fracture resistance mean values for all groups

\section{DISCUSSION}

Endodontic treatment and access repair in allceramic crowns is an inevitable event as such restorations became widely used. A functional and esthetic repair has many advantages over time consuming and expensive remakes of ceramic restorations. ${ }^{(12)}$ A survey of 543 dental practitioners (prosthodontists, endodontists, and general practitioners) showed that $72 \%$ choose to gain access through existing crowns and maintain it as a permanent restoration rather than removing the crown or placing a temporary crown. ${ }^{(19)}$

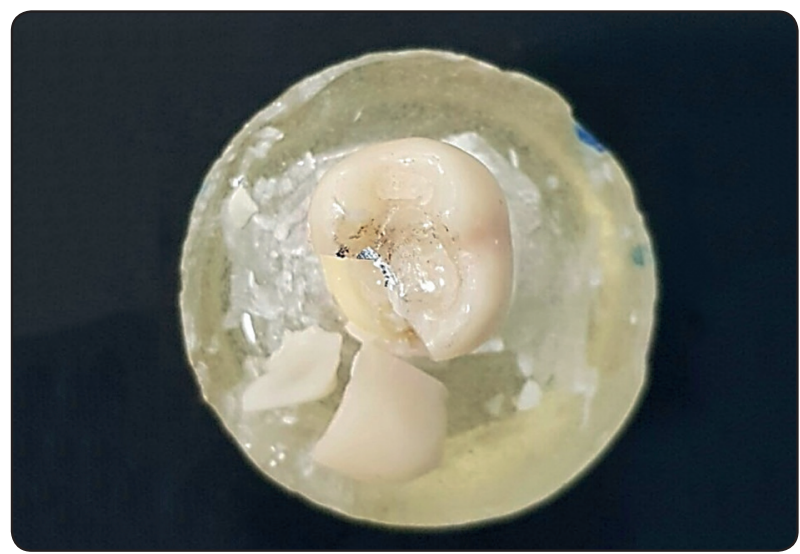

Fig. (5) Fractured crown with intact tooth structure

Therefore, the main interest of this study was directed towards evaluating the fracture resistance of endodontically accessed hybrid ceramic crowns repaired with direct composite compared to those repaired with ceramic plugs.

Natural teeth were used in this study as their modulus of elasticity, bonding characteristics and strength mimic the clinical situation better than would metal or acrylic resin teeth. ${ }^{(12)}$ A lower molar was selected as this tooth was determined to be treated most often with endodontic therapy. ${ }^{(20)}$ 
For the purpose of standardization, a Biogeneric copy mode in the Cerec Premium software 4.4 was used so that each crown was designed and milled to be an exact replica of the unprepared anatomy. All teeth were prepared following the clinically established preparation criteria for all ceramic crowns ${ }^{(21)}$ with the aid of a special milling machine to ensure standardization.

Although there is a great variety of dental ceramic materials and a large selection of composite resin materials in the market, polymer infiltratedceramic-network material (Vita Enamic) was used in this study. Vita Enamic has a dual-network structure where the dominant ceramic network is reinforced by a polymer network and thus combining the positive characteristics of ceramic and composite and offering an integrated crack stop function. In addition, the polymer network offers an outstanding absorption of intraoral forces rendering it suitable for posterior crown restorations. Also, it has an elasticity close to dentin and good bonding characteristics. $^{(22,23)}$

A strict adherence to the bonding and luting protocols for the ceramic material used was followed to eliminate any variables during bonding and luting procedures. $^{(24,25)}$ Crowns were cemented using Rely X Ultimate dual cure adhesive resin cement. It is generally agreed that resin cement acts as an inherent buffering layer that is capable of absorbing forces during load application and thus leading to increased fracture resistance values. ${ }^{26,27)}$

Endodontic access openings through all ceramic restorations may initiate microcracks which could propagate with time and cause fracture. Considering these challenges, access openings were done with a diamond rotary cutting instrument in a high speed hand piece with heavy water spray in accordance with Sutherland et al ${ }^{(28)}$ Haselton et al ${ }^{(29)}$ and Oden et $\mathrm{al}^{(30)}$ in order to avoid the vibration of low speed hand pieces which may produce cracks at the ceramic margins. Diamonds were the instrument of choice rather than carbide burs because they are more suited for ceramics as the mechanism of grinding leads to a smoother cut with less vibration. ${ }^{(31,32)}$ In addition, a conservative access cavity preparation was done with minimal lingual extension as the lingual cusps of mandibular molars fractured more often than the buccal cusps by ratio $2: 1$. $^{(33)}$

The decision to repair the access opening or replace the existing crown was based on the extent of visible micro-cracks induced during access preparation.

Ideally, repair of access cavities should be done with a material that provides a leak proof seal. Unfortunately all materials that are used leak to some extent. ${ }^{(12)}$ In addition, the repair material should restore the strength of the accessed restoration. Therefore the idea of our study was to use a ceramic plug to repair the access cavity in an attempt to provide a good seal and improve the fracture resistance of the repaired accessed crown. The ceramic plug was fabricated from Vita Enamic which was the same material of the accessed crown. This hybrid material is already at full strength and can be inserted immediately after milling and thus time saving. ${ }^{(23)}$

Thermo mechanical aging was performed for all the tested specimens in a chewing simulator in order to mimic the intraoral conditions. The test was repeated for 150,000 cycles to simulate a 1 year of clinical service. ${ }^{(34)}$

Fracture resistance evaluation was chosen in this study as it is one of the critical factors that determine the success and longevity of the restoration. The load to fracture in this study was applied vertically similarly to the technique used by Sedgley and Messer ${ }^{(35)}$ to test the brittleness of endodontically treated teeth.

Results of several studies reported that the mean maximum masticatory forces encountered in the molar region are $847 \mathrm{~N}$ for men and $597 \mathrm{~N}$ for women. ${ }^{(36,37)}$ 
Regarding the results of our study, the highest mean fracture resistance values were recorded for group I "Teeth restored with full coverage Enamic crowns" $(2027.64 \pm 294.24 \mathrm{~N})$ followed by group III "Teeth restored with full coverage Enamic crowns, endodontically treated and repaired with bonded Enamic plugs" (1784.26 $\pm 72.15 \mathrm{~N})$ while the lowest mean fracture resistance values were recorded for group II "Teeth restored with full coverage Enamic crowns, endodontically treated and repaired with direct composite" (1494.95 \pm 46.95 $\mathrm{N})$. The difference between groups was statistically significant as indicated by ANOVA test.

As shown, the mean fracture resistance values for the different groups were beyond the mean reported maximum masticatory forces. Therefore, it can be assumed that all the tested specimens in the three groups could withstand the maximum intraoral posterior masticatory forces.

The higher fracture resistance values of crowns in group I over those in the other two groups may be attributed to being intact and thus providing a good distribution of stresses generated by axial loading. ${ }^{(12)}$ It might be also related to the fact that endodontic access cavities made through hybrid ceramic crowns may create a variety of flaws some of each may have the potential to cause a reduction of strength. ${ }^{(13)}$

This was in accordance with Shaker and El Demellawy ${ }^{(12)}$ and Wood et al. ${ }^{(13)}$ who declared that access cavities through all ceramic crowns resulted in a significant loss of strength.

Regarding the repair protocol, the results obtained in this study showed that crowns repaired with bonded Enamic plugs had higher fracture resistance values compared to those repaired with direct composite. This may be linked to the drawbacks of the composite compared to ceramics namely the lower strength values and polymerization shrinkage.

It may also be related to the plug material (Vita Enamic) being a shock absorber with a modulus of elasticity close to that of dentin and good bonding characteristics. ${ }^{(22)}$
Another possible explanation is the similarity between the plug material and the crown material which may provide a monoblock effect. When subjected to occlusal loads, the whole monoblock system will allow even distribution of stresses and will deform uniformly decreasing the stresses transferred to the vulnerable tooth structure. ${ }^{(38,39)}$

However, when post Hoc test was performed, no significant difference was found between groups I and III which indicates the reliability of the ceramic plug to restore endodontically accessed crowns.

Also the post Hoc test revealed that there was no significant difference between groups II and III which makes the use of composite still an acceptable repair protocol.

According to the previous discussion, our results opposed the null hypothesis, where the bonded ceramic plug increased the fracture resistance values of repaired endodontically accessed hybrid ceramic crowns compared to direct composite resin.

\section{RECOMMENDATIONS}

Although our study revealed the possibility of using a ceramic plug as a repair protocol for endodontically accessed hybrid ceramic crowns, however, clinical studies are required to confirm our finding and provide definitive results about the longevity of this repair protocol.

\section{CONCLUSIONS}

Within the limitations of this study, the following conclusions were drawn;

1- The endodontically accessed hybrid ceramic crowns repaired with the novel protocol (ceramic plug) recorded high fracture resistance values.

2- The use of ceramic plug is a feasible alternative to the currently used composite repair protocol. 


\section{REFERENCES}

1. Christensen, G.J.: Too many crowns? J. Am. Dent. Assoc. 144 (2013) 1174-1176.

2. Edelhoff, D. and Sorensen, J.A.: Tooth structure removal associated with various preparation designs for anterior teeth. J. Prosthet. Dent. 87 (2002) 503-509.

3. Goodacre, C.J. and Spolnik, K.J.: The prosthodontic management of endodontically treated teeth: a literature review. Part I. Success and failure data treatment concepts. J. Prosthodont. 3 (1994) 243-250.

4. Christensen, G.J.: Tooth preparation and pulp degeneration. J. Am. Dent. Assoc. 128 (1997) 353-354.

5. Christensen, G.J.: How to kill a tooth. J. Am. Dent. Assoc. 136 (2005) 1711-1713.

6. Goodacre, C.J. ; Bernal, G.; Rungcharassaeng, K. and Kan, J.Y.: Clinical complications in fixed prosthodontics, J. Prosthet. Dent. 90 (2003) 31-41.

7. Pjetursson, B.E.; Sailer, I.; Zwahlen, M. and Hämmerle, C.H.: A systematic review of the survival and complication rates of all-ceramic and metal-ceramic reconstructions after an observation period of at least 3 years. Part I: single crowns. Clin. Oral Implan. Res. 18 (Suppl. 3) (2007) 73-85.

8. Ortorp, A. ; Kihl, M.L. and Carlsson, G.E.: A 5-year retrospective study of survival of zirconia single crowns fitted in a private clinical setting. J. Dent. 40 (2012) 527-530.

9. Rinke, S.; Lange, K. ; Roediger, M. and Gersdorff, N.: Risk factors for technical and biological complications with zirconia single crowns, Clin. Oral Investig. 19 (2015) 1999-2006.

10. Burke, F.J.T. and Lucarotti, P.S.K.: Re-intervention on crowns: what comes next? J. Dent. 37 (2009) 25-30.

11. Saunders, W.P. and Saunders, E.M.: Prevalence of peri radicular periodontitis associated with crowned teeth in an adult Scottish subpopulation, Brit. Dent. J. 185 (1998) $137-140$.

12. Shaker,A. and El Demellawy, M.A.: Effect of Endodontic access on the strength of an existing all-ceramic crown. Egy. Dent. J. 57 (2011) 3055-3063.

13. Wood, K.C.; Berzins, D.W.; Luo, Q.; Thompson, G.A.; Toth, J.M. and Nagy, W.W.: Resistance to fracture of two all-ceramic crown materials following endodontic access $\mathrm{J}$ Prosthet Dent 95 (2006) 33-41.
14. Thompson, J.Y.; Anusavice, K.J.; Naman,A. and Morris, H.F.: Fracture surface characterization of clinically failed all-ceramic crowns, J. Dent. Res.73(1994) 1824-1832.

15. Rekow, D. and Thompson, V.P.: Engineering long term clinical success of advanced ceramic prostheses, J. Mater. Sci-Mater. Med. 18 (2007) 47-56.

16. Gorman, C.M.; Ray, N.J. and Burke, F.M.: The effect of endodontic access on all-ceramic crowns: A systematic review of in vitro studies. J. Dent. 53 (2016) 22-29.

17. Trautmann, G.; Gutmann, J.L.; Nunn, M.E.; Witherspoon, D.E. and Shulman, J.D.: Restoring teeth that are endodontically treated through existing crowns. PartII: survey of restorative materials commonly used, Quintessence Int. 31 (2000) 719-728.

18. Goldman, M.; Laosonthorn, P. and White, R.R.: Micro leakage-full crowns and the dental pulp. J Endod. 18 (1992) 473-475.

19. Trautmann, G.; Gutmann, J.L.; Nunn, M.E.; Witherspoon, D.E. and Shulman, J.D.: Restoring teeth that are endodontically treated through existing crowns. Part I: Survey of pulpal status on access, Quintessence Int. 31 (2000) 713-718.

20. Wayman, B.E.; Patten, J.A. and Dazey, S.E.: Relative frequency of teeth needing endodontic treatment in 3350 consecutive endodontic patients. J Endod. 20 (1994) 399-401.

21. Bindl A. and Mörmann, W.H.: Clinical evaluation of adhesively placed Cerec Endocrowns after 2 years Preliminary results. J. Adhes. Dent. 1 (1999) 255-265.

22. Dirxen, C.; Blunck, U. and Preissner, S.: Clinical Performance of a New Biomimetic Double Network Material. Open Dent. J. 7 (2013) 118-122.

23. Vita Enamic, The concept. Vita Zahnfabrik, July 2016.

24. Bindl, A.; Richter, B. and Mörmann, W.H.: Survival of ceramic computer-aided design/manufacturing crowns bonded to preparations with reduced macroretention geometry. Int. J. Prosthodont. 18 (2005) 219-224.

25. Goto, Y.; Nicholls, J.I.; Phillips, K.M. and Junge, T.: Fatigue resistance of endodontically treated teeth restored with three dowel-and-core systems. J. Prosthet. Dent. 93 (2007) 45-50.

26. Gu, H. and Kern, M.: Marginal discrepancies and leakage of all ceramic crowns: influence of luting agents and aging conditions. Int. J. Prosthodont. 16 (2003) 109-116. 
27. Blatz, M.B.; Sadan, A. and Kern, M.: Resin-ceramic bonding: A review of the literature. J. Prosthet. Dent. 89 (2003) 268-274

28. Sutherland, J.K.; Teplitsky, P.E. and Moulding, M.B.: Endodontic access of all-ceramic crowns. J. Prosthet. Dent. 61 (1989) 146-149

29. Haselton, D.R.; Lloyd, P.M. and Johnson, W.T.: A comparison of the effects of two burs on endodontic access in all-ceramic high leucite crowns. Oral Surg Oral Med Oral Pathol Oral Radiol Endod. 89 (2000) 486-492.

30. Oden, A.; Andersson, M.; Krystek-Ondracek, I. and Magnusson, D.: Five-year clinical evaluation of Procera AllCeram crowns. J Prosthet Dent. 80 (1998) 450-456.

31. Qeblawi, D.; Hill, T. and Chlosta, K.: The effect of endodontic access preparation on the failure load of lithium disilicate glass-ceramic restorations, J. Prosthet. Dent. 106 (2011) 329-335.

32. Bompolaki, D.; Konogiorgos, E.; Wilson, J.B. and Nagy, W.W.: Fracture resistance of lithium disilicate restorations after endodontic access preparation: an in vitro study J. Prosthet. Dent. 114 (2015) 580-586

33. Eakle, W.S.; Maxwell, E.H. and Braly, B.V.: Fractures of posterior teeth in adults. J Am Dent Assoc. 112(2) (1986) 215-218
34. Nawafleh, N.; Hatamleh, M.; Elshiyab, S. \& Mack, F.:Lithium Disilicate Restorations Fatigue Testing Parameters: A Systematic Review. J Prosthodont. 25(2) (2016) 116-126

35. Sedgley, C.M. and Messer, H.H.: Are endodontically treated teeth more brittle? J. Endodont. 18 (1992) 332-335.

36. Haraldson, T.; Carlsson, G.E. and Ingervall, B.: Functional state, bite force and postural muscle activity in patients with osseointegrated oral implant bridges. Acta Odont. Scand. 37 (1979) 195-206.

37. Magne, P. and Knezevic, A.: Stimulated fatigue resistance of composite resin versus porcelain CAD/CAM overlay restorations on endodontically treated molars. Quint. Int. 40 (2) (2009) 125-133.

38. Chang, C.Y.; Kuo, J.S.; Lin, Y.S. and Chang, Y.H.: Fracture resistance and failure modes of CEREC endocrowns and conventional post and core-supported CEREC crowns. J. Dent. Sci. 4 (3) (2009) 110-117.

39. Lin, C.L.; Chang, Y.H.; Chang, C.Y.; Pai, C.A. and Huang, S. F.: Finite element and Weibull analyses to estimate failure risks in the ceramic endocrown and classical crown for endodontically treated maxillary premolar. Eur. J. Oral Sci. 118 (2010) 87-93. 